\section{Percutaneous cholecystostomy FOR ACUTE CHOLECYSTITIS: NOT SO SAFE AFTER ALL}

I read with interest the paper by Molavi and colleagues, ${ }^{1}$ entitled "Clinical and operative outcomes of patients with acute cholecystitis who are treated initially with imageguided cholecystostomy." Any examination of the use of percutaneous cholecystostomy and resultant outcomes is appreciated. However, a large, multi-centre, randomized controlled trial (the CHOCOLATE trial), referenced by Molavi and colleagues, has been conducted to address whether percutaneous cholecystostomy had advantages over early laparoscopic cholecystectomy for high-risk patients (APACHE-II score 7-15) with acute cholecystitis. What is omitted from Molavi and colleagues' discussion is the fact that in 2016 the CHOCOLATE trial had to be terminated prematurely after interim analysis had raised concerns about the negative effects of percutaneous cholecystostomy. There was a markedly significantly lower rate of the primary composite endpoint (major complications, readmission, reintervention and death) in high-risk patients treated with early laparoscopic cholecystectomy compared with percutaneous cholecystostomy. Consequently, I question the characterization by Molavi and colleagues of percutaneous cholecystostomy as a safe procedure with low morbidity (despite not reporting all morbidities or outcomes in their review). The question is: safe compared to what?

\section{Scott Cowie, MD}

Affiliation: From the Langley Memorial Hospital, Langley, BC.

Competing interests: None declared. DOI: $10.1503 /$ cjs. 1861041

\section{References}

1. Molavi I, Schellenberg A, Christian F. Clinical and operative outcomes of patients with acute cholecystitis who are treated initially with image-guided cholecystostomy. Can F Surg 2018;61:195-9.

\section{AUtHOR RESPONSE}

We thank Dr. Cowie for his interest in our article and for his letter.

The question Dr. Cowie poses at the end of his letter is precisely what our article sets out to answer, and we believe that our article in fact does support the conclusion that laparoscopic cholecystectomy may in fact be a safer and better option than percutaneous tube cholecystostomy (PCT) for the treatment of acute cholecystitis in patients who have significant complicating comorbidities.

Our study shows quite clearly that only $31.4 \%$ of our study cohort with cholecystostomy tubes in place returned for elective laparoscopic cholecystectomy and that, in this group, among those who underwent laparoscopic cholecystectomy, the conversion to open cholecystectomy was unacceptably high (18\%). In addition, there were significant readmission rates for tube complications (15\%) and for recurrent cholecystitis after tube removal (7.1\%).

Our paper therefore makes the case that in fact the widespread practice of percutaneous tube cholecystostomy (PCT) in this subgroup of patients with acute cholecystitis needs a reappraisal and that tube cholecystostomy is not so safe after all.

With regard to Dr. Cowie's question about the perceived safety of PCT in the surgical literature and surgical community, references 11-16 in our article as well as a more recent study ${ }^{1}$ support this view.

Although the CHOCOLATE trial results have not been published, we are well aware of its commendable goals: the trial was conceived as a prospective study of PCT versus laparoscopic cholecystectomy, and its study protocol was released in 2012. ${ }^{2}$ Subsequently, the trial was indeed terminated since the PCT group had a significantly higher mortality; again, this was not published in a peer-reviewed journal, but in a monograph. ${ }^{3}$ We would seriously question the conclusions of the CHOCOLATE trial, in which there was a mortality of $68 \%$ in their PCT patient group and $15 \%$ in the laparoscopic cholecystectomy group; Canadian and American general surgeons cannot relate to the high mortality in both these subgroups.

Nevertheless, our study shows that PCT as a procedure of first choice in the treatment of high-risk patients with acute cholecystitis is in need of an urgent reappraisal.

\section{Francis Christian, MD}

Affiliation: From the Department of General Surgery, University of Saskatchewan, Saskatoon, Sask.

Competing interests: None declared. DOI: $10.1503 /$ cjs. 1861042

\section{References}

1. Chih-Hung W, Wu C-Y, Yang J, et al. Long-term outcomes of patients with acute cholecystitis after successful percutaneous cholecystostomy treatment and the risk factors for recurrence: a decade experience at a single center. PLoS One 2016;11: :e0148017.

2. Kortram K, Kirsten K, van Ramshorst B, et al. Acute cholecystitis in high risk surgical patients: percutaneous cholecystostomy versus laparoscopic cholecystectomy ]. [CHOCOLATE trial]. [: Study protocol for a randomized controlled trial. Trials 2012;13:7.

3. Loozen CS, van Santvoort HC. Laparoscopic cholecystectomy versus percutaneous catheter drainage for high-risk patients with acute cholecystitis in: C.S. Loozen, "Optimal Treatment of Acute Cholecystitis," Utrecht University, The Netherlands. 2017: 133-153. 